\title{
Structural Stability and Transformation of Aligned C60 and C70 Fullerenes in Double-Wall and Triple-Wall Carbon Nanotube Peapods
}

Guoqing Ning, ${ }^{a}$ Naoki Kishi, ${ }^{b}$ Haruya Okimoto, ${ }^{b}$ Masahiro Shiraishi, ${ }^{b}$ Toshiki Sugai,${ }^{b}$ Hisanori Shinohara ${ }^{*}, a, b$

${ }^{a}$ CREST, Japan Science and Technology Agency, c/o Department of Chemistry, Nagoya University, Nagoya 464-8602, Japan.

${ }^{\mathrm{b}}$ Department of Chemistry and Institute for Advanced Research, Nagoya University, Nagoya 464-8602, Japan.

*Corresponding author. E-mail: noris@cc.nagoya-u.ac.jp

\section{Method for TEM measurement and errors}

a) Method for measurement
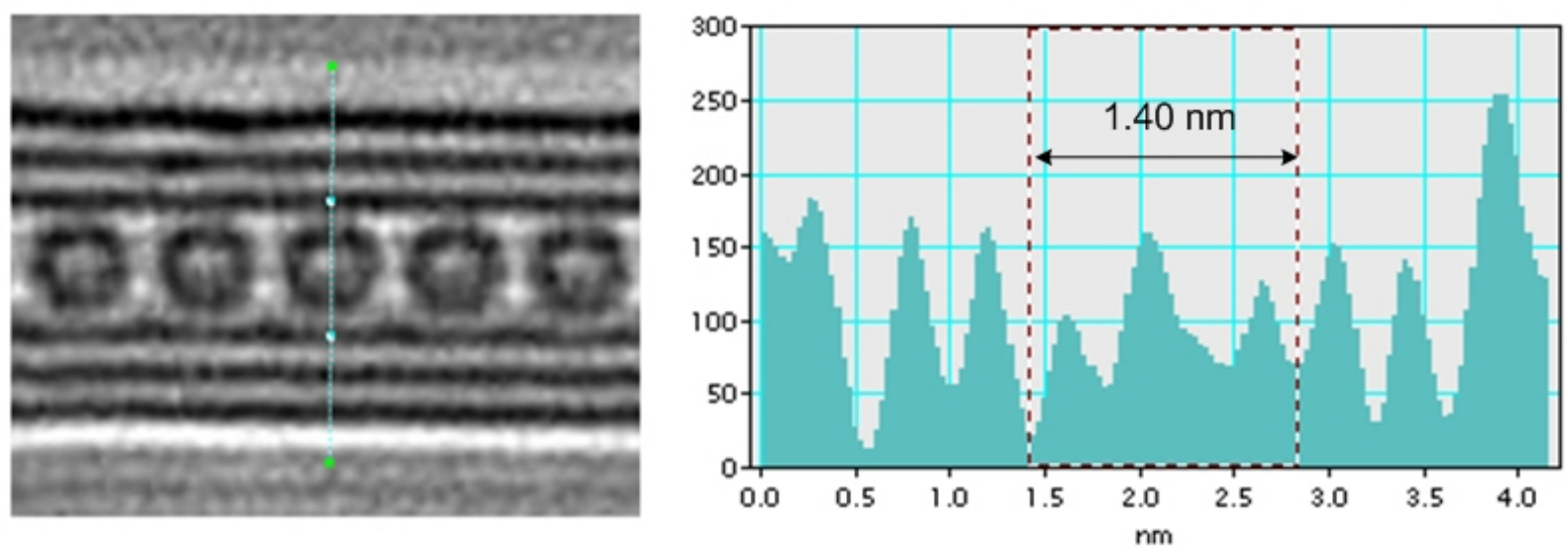

In TEM images, fuscous lines indicate the positions and shapes of different species. The central positions of the lines were used to make the measurement of TEM images, as shown in the above image.

b) Errors of measurement: $\pm 0.03-0.04 \mathrm{~nm}$ 


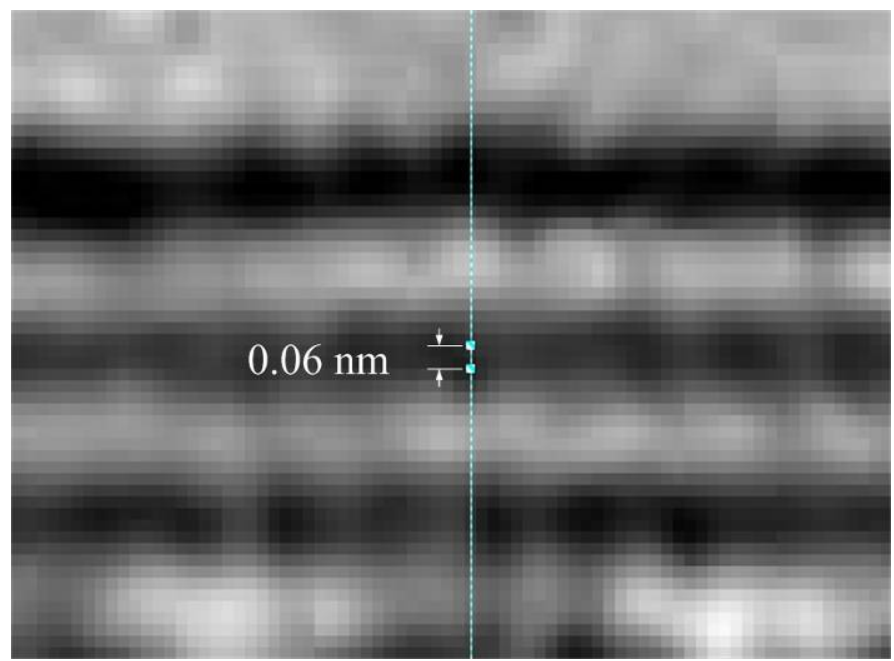

The black lines corresponding to the graphite layers of a TWNT in Figure 2c are shown in the above image. Two points on the black line with a distance of $0.06 \mathrm{~nm}$ can be well distinguished (they stay in the different sides of the central position of the line). Thus the error for the measurement in Figure 2c is estimated as $\pm 0.03 \mathrm{~nm}$.

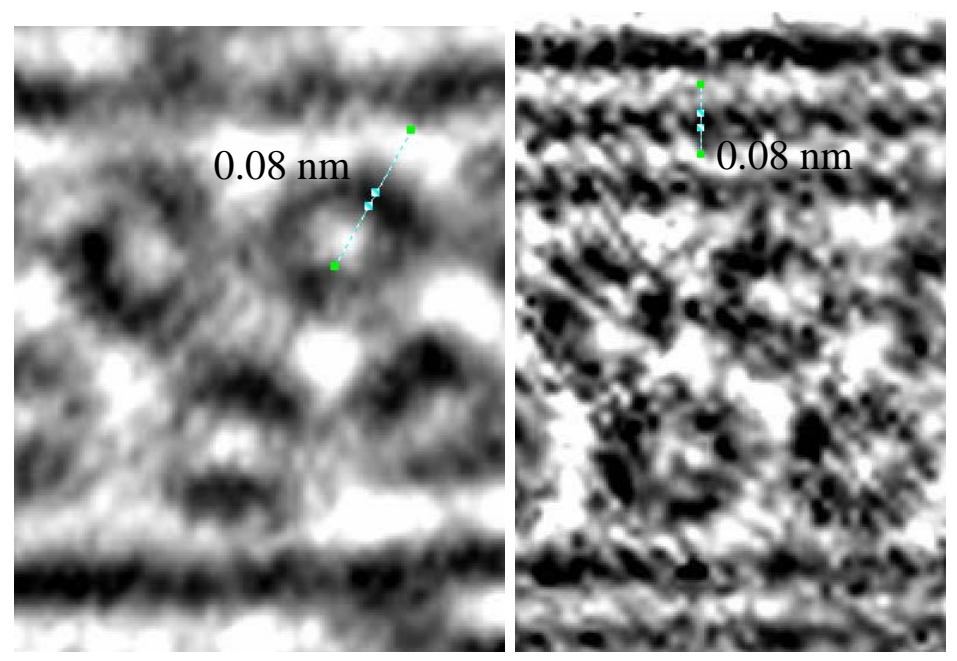

The error for the measurement in Figure 3 is estimated as $\pm 0.04 \mathrm{~nm}$. 